\title{
Parastomal Hernia as a Risk Factor for Ileal Conduit Fistulae
}

\author{
Thomas Andrew Alexander Skinnner, Richard Watson Norman
}

Department of Urology, Dalhousie University, Halifax, Canada.

Email: richard.norman@dal.ca

Received January 29 ${ }^{\text {th }}, 2011$; revised April $16^{\text {th }}$, 2011; accepted May $11^{\text {th }}, 2011$.

\begin{abstract}
Purpose: to review potential risk factors for the development of ileal conduit fistulae. Methods: two patients were identified who had a remote history of an ileal conduit and who formed a fistula from the conduit-one to the small bowel and one to the skin. Their presentation, management and outcomes are described. Results: Both patients had parastomal hernias as the likely cause of their fistula formation. Discussion: parastomal herniation may contribute to fistula formation due to a strangulated ischemic pressure necrosis of the adjacent ileal conduit and/or bowel.
\end{abstract}

Keywords: Fistula, Ileal Conduit, Parastomal Hernia, Urinary Diversion

\section{Introduction}

Advancements in surgical procedures can lead to new and complex problems. Over the past 40 years, radical cystectomy has become the treatment of choice for invasive or aggressive bladder cancers, as well as for other bladder disorders. The development of this procedure has lead to a variety of urine management systems designed to replace the bladder's storage and voiding functions. Bladder substitution with an ileal conduit was pioneered by Eugene M. Bricker over half a century ago and remains a popular technique today [1,2]. This method of urinary diversion requires a relatively simple surgical procedure, has low complications rates and is associated with good patient quality of life [2,3]. It does, however, open the door to a number of potential problems.

Complications following the creation of a Bricker conduit may be separated into early and late events. Early complications are most commonly visceral, including gastrointestinal or urinary fistulae and intestinal ileus. Delayed complications tend to consist of urological or parietal issues including acute pyelonephritis, ureteroileal stricturing, urolithiasis, incisional hernias, parastomal hernias and stricturing of the stoma [4]. Stomal complications are generally regarded as the commonest problem associated with ileal conduits with parastomal hernia occurring most frequently [4-6]. Identified risk factors for the development of parastomal hernia include advanced age, obesity, steroid use, chronic cough and malnutrition [4]. Less common is the occurrence of ileal conduit fistulae, which can cause serious morbidity often requiring surgical intervention [7]. Risk factors for development of fistulae are not well understood.

We report two patients with a remote history of an ileal conduit who developed parastomal hernias and went on to form a fistula from the conduit-one to the small bowel and one to the skin. We suggest that both developed as secondary complications of parastomal hernia formation, which may explain their late presentation.

\section{Case \# 1}

Forty-five years earlier, this 77 year old woman had marked ureteric dilatation and renal scarring and underwent partial cystectomy and bilateral ureteric re-implantation. Three years later she was converted to an ileal conduit urinary diversion due to voiding dysfunction, intolerance of a urethral catheter and upper tract deterioration. Five years after she developed stomal stenosis treated by dilatation and then revision. In the same year, she had small bowel resection for obstruction secondary to adhesions with gangrenous bowel. Four years subsequently she had an elective cholecystectomy and further revision of her stoma because of re-stenosis and recurrent symptomatic urinary tract infections. She continued to have intermittent problems with stomal stenosis managed by daily finger/catheter dilatation and eventually the stoma was revised again 38 and 39 years after her original diversion. She was first noted to have parastomal hernia 1 year later. A year after that she developed a 
spontaneous mid-left ureteric leak which healed with percutaneous nephrostomy drainage and antegrade stenting and 1 year after that an asymptomatic parastomal hernia was noted to contain incarcerated small bowel (Figure 1). She was reviewed by general surgery and it was decided to watch her expectantly. One year later she developed a small bowel to conduit fistula confirmed by computerized tomography. Serum creatinine varied between $200-300 \mathrm{umol} / \mathrm{L}$. A trial of TPN was unsuccessful. She underwent a complicated laparotomy, lysis of numerous small bowel adhesions, excision of the scarred ileal loop and resection of several segments of small bowel. It was felt folly to attempt to reconstruct a new conduit in view of the state of the bowel and ureters, and both ureters were clipped off. The hernia was repaired a with a large Surgisis mesh. Bilateral percutaneous nephrostomy drainage was established post-operatively. She has never felt better and her recent serum creatinine is $136 \mathrm{umol} / \mathrm{L}$.

\section{Case \#2}

This 70 year old diabetic woman had an ileal conduit urinary diversion 12 years earlier because of progressive bilateral hydronephrosis, impaired bladder emptying and recurrent funguria. One year later she was noted to have a parastomal hernia which was watched expectantly. Three years later she developed a partial small bowel obstruction, urosepsis and bilateral hydronephrosis requiring bilateral nephrostomy tubes and ICU support. She formed an enterocutaneous fistula that closed spontaneously after total parenteral nutrition. She did well although her parastomal hernia continued to enlarge and was seen on a computerized tomographic scan (CT) 5 years later. After another 2 years, she developed a small

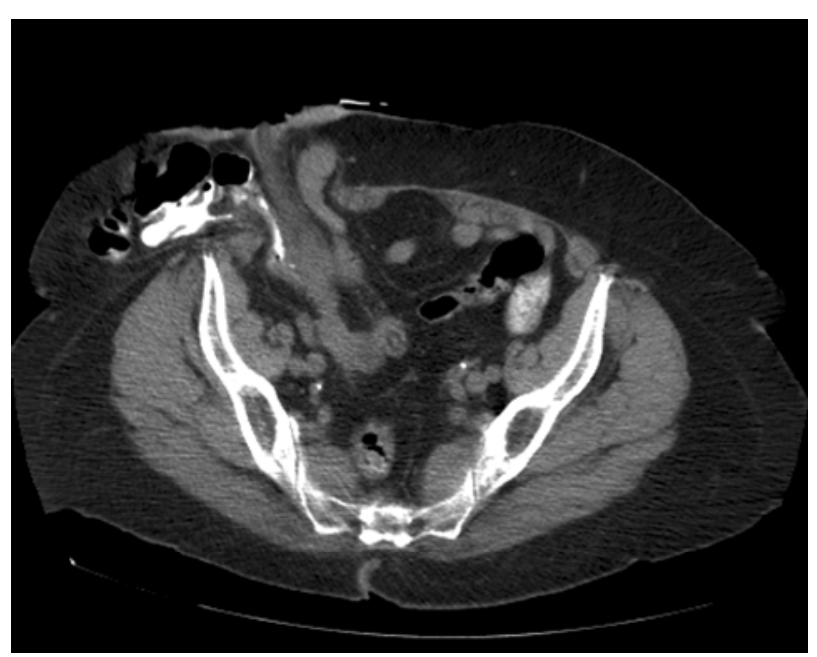

Figure 1. Parastomal herniation of incarcerated bowel is seen in this CT scan of case \#1. subcutaneous abscess that started putting out urine. Loopogram showed the connection to the skin. A foley catheter was inserted into the ileal loop and the fistula dried up in 10 days. She is doing well 2 year later but the parastomal hernia continues to grow and it is regularly evaluated by general surgery (Figure 2). The current feelings are that the risks of surgery outweigh the potential benefits.

\section{Discussion}

Ileal conduit fistulae are largely restricted to old case reports [8]. These fistulae may present in a variety of ways - transcutaneous urine leakage, passage of gas or gastrointestinal contents through the ileal stoma, severe intractable diarrhea, and refractory pyelonephritis $[9,10]$. Although some suggest that fistula formation is predominantly an early complication following urinary diversion surgery, we and others report examples of ileal conduit fistulae occurring many years after they were created $[8,9]$. These include both conduit-cutaneous and conduit-enteric fistulae $[9,10]$. Although the mechanism of fistula formation in unclear potential risk factors include prior radiation, urinary stones, chronic inflammation, diabetes mellitus and stomal structuring [10]. Specific case reports have also provided evidence that local tumors or abscess formation may play a role [9]. We propose that parastomal herniation contributed to fistula formation in both of our patients due to a strangulated ischemic pressure necrosis of the adjacent conduit and/or bowel.

\section{Acknowledgements}

Neither author has any industrial link or affiliation related to the subject matter in this manuscript.

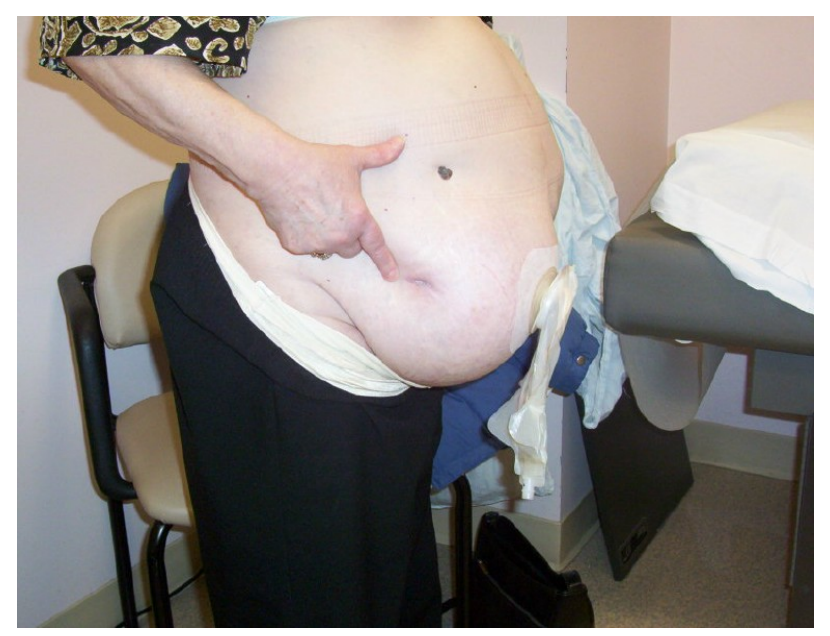

Figure 2. The patient described as case \#2 points to the site of her previous conduit-cutaneous fistula. The large parastomal hernia is seen adjacent to the appliance. 


\section{REFERENCES}

[1] E. M. Bricker, "Bladder Substitution after Pelvic Evisceration,” Surgical Clinics of North America, Vol. 30, No. 5, 1950, pp. 1511-1521.

[2] W. J. Yang, K. S. Cho, K. H. Rha, et al., "Long-Term Effects of Ileal Conduit Urinary Diversion on Upper Urinary Tract in Bladder Cancer,” Urology, Vol. 68, No. 2, 2006, pp. 324-327. doi:10.1016/j.urology.2006.02.015

[3] P. S. Frich, C. A. Kvestad and A. Angelsen, "Outcome and Quality of Life in Patients Operated on with Radical Cystectomy and Three Different Urinary Diversion Techniques," Scandinavian Journal of Urology and Nephrology, Vol. 43, No. 1, 2009, pp. 37-41. doi:10.1080/00365590802473198

[4] S. B. Farnham and M. S. Cookson, "Surgical Complications of Urinary Diversion," World Journal of Urology, Vol. 22, No. 3, 2004, pp. 157-167. doi:10.1007/s00345-004-0429-5

[5] E. Kouba, M. Sands, A. Lentz, E. Wallen and R. S. Pruthi, "Incidence and Risk Factors of Stomal Complications in Patients Undergoing Cystectomy with Ileal Conduit Urinary Diversion for Bladder Cancer,” Journal of Urology, Vol. 178, No. 3, 2007, pp. 950-954.

doi:10.1016/j.juro.2007.05.028
[6] D. N. Wood, S. E. Allen, M. Hussain, T. J. Greenwell and P. J. Shah, "Stomal Complications of Ileal Conduits are Significantly Higher When Formed in Women with Intractable Urinary Incontinence,” Journal of Urology, Vol. 172, No. 6, 2004, pp. 2300-2303. doi:10.1097/01.ju.0000141140.56022.7a

[7] E. C. Kauffman, C. K. Ng, M. M. Lee, et al., "Critical Analysis of Complications after Robotic-Assisted Radical Cystectomy with Identification of Preoperative and Operative Risk Factors,” British Journal of Urology International, Vol. 105, No. 4, 2010, pp. 520-527.

[8] L. Msezane, W. S. Reynolds, R. Mhapsekar, G. Gerber and G. Steinberg, "Open Surgical Repair of Ureteral Strictures and Fistulas Following Radical Cystectomy and Urinary Diversion,” Journal of Urology, Vol. 179, No. 4, 2008, pp. 1428-1431. doi:10.1016/j.juro.2007.11.083

[9] B. Rumstadt, B. Klein and D. Schilling, "Fistula between Ileal Conduit and Caecum: Unusual Presentation of a Colonic Carcinoma," Australian and New Zealand Journal of Surgery, Vol. 80, No. 6, 2010, p. 466. doi:10.1111/j.1445-2197.2009.05194.X

[10] N. Hampel and A. Zabbo, "Parastomal Ileal Conduit-Enteric Fistula,” Journal of Urology, Vol. 134, No. 5, 1985, pp. 956-957. 